\title{
Biofilms associated with bowel necrosis: A newly recognised phenomenon in infants
}

\author{
G Brisighelli, S Cox, A Theron, K Pillay, H Rode \\ Dr Giulia Brisighelli is a registrar in paediatric surgery at the UOC Chirurgia Pediatrica, Fondazione IRCCS Ca' Granda Ospedale Maggiore \\ Policlinico, Milan, Italy. She worked as supernumerary registrar in paediatric surgery at Red Cross War Memorial Children's Hospital, Cape Town, \\ South Africa. Dr Sharon Cox is the Head of the Clinical Unit in the Department of Paediatric Surgery at Red Cross War Memorial Children's \\ Hospital and is affiliated to the Faculty of Health Sciences at the University of Cape Town. Dr André Theron is a senior paediatric surgical registrar \\ working at Red Cross War Memorial Children's Hospital. He has a keen interest in neonatal surgery. Associate Professor Komala Pillay is a paediatric \\ pathologist at the National Health Laboratory Service and University of Cape Town, based at Red Cross War Memorial Children's Hospital. Prof. \\ Heinz Rode is Emeritus Professor of Paediatric Surgery, based at Red Cross War Memorial Children's Hospital and affiliated to the Faculty of Health \\ Sciences at the University of Cape Town.
}

Corresponding author: S Cox (sharon.cox@uct.ac.za)

Background. A biofilm is defined as a collection of organisms attached to a surface and surrounded by a matrix.

Objective. To present three cases in which bowel necrosis coexisted with biofilm.

Methods. The medical records, bacteriological findings and tissue biopsies from three infants with bowel necrosis who subsequently died from sepsis were analysed. Tissue sent for histological evaluation was prepared for light microscopy. Haematoxylin and eosin (H\&E), Sandiford and Alcian blue/periodic acid Schiff (ABPAS) stains were performed. Tissue samples were ex-waxed for electron microscopy in one case.

Results. The three patients described all had necrotic bowel at laparotomy, all cultured Klebsiella pneumoniae from peritoneal pus swabs, and all died despite appropriate antibiotics. All specimens showed varying degrees of bowel necrosis and an organising acute peritoneal reaction. In addition, all showed colonies of Gram-negative bacteria within a mucopolysaccharide matrix.

Conclusions. The identification of biofilms in necrotic bowel has raised questions regarding their clinical implications. Further studies are needed to evaluate all resected necrotic bowel for biofilms and the clinical implications of this finding.

S Afr Med J 2016;106(4):345-347. DOI:10.7196/SAMJ.2016.v106i4.10425

The term biofilm has been used to describe a well-organised microbial community enmeshed in a polymeric, carbohydrate-rich extracellular matrix and adhering to an inanimate or living surface. ${ }^{[1]}$ The organisms within it become highly resistant to antibiotic therapy and to the immune system. ${ }^{[2]}$ The reasons for this are complex and are related to the inability of antibiotics to diffuse through the biofilm, to changes in the organisms resulting from the increasing hypoxia within the biofilm, and to the development of persistent resistant cells thought to be a mechanism of survival by the micro-organisms. ${ }^{[3]}$ Although biofilms have been detected in several mucosal locations, their ability to trigger human disease is still a matter of active investigation. ${ }^{[4]}$ The pathogenic role of biofilms has been established for oral infections, chronic wounds, indwelling medical and surgical devices, and implants. ${ }^{[1,3,5]}$ Currently, the link between gastrointestinal infections and biofilms in infants is less clear. ${ }^{[6]}$ We encountered three infants with necrotic small bowel in whom special staining techniques on histological specimens of their resected bowel revealed an abundance of Klebsiella-associated intramural biofilm formations. This phenomenon has not been described before in necrotic bowel in infants and may be of significance in the pathogenesis of bowel necrosis and the medical and surgical management thereof, and have implications in the understanding of the disease process.

The objective of this article is to present three cases in which bowel necrosis coexisted with biofilm.

\section{Methods}

The medical records, bacteriological findings and tissue biopsies from three infants who underwent laparotomy for volvulus, necrotising enterocolitis (NEC) and spontaneous intestinal perforation, and who subsequently died from sepsis, from March 2014 to March 2015 at Red Cross War Memorial Children's Hospital, Cape Town, South Africa, were analysed. Tissue sent for histological evaluation was prepared for light microscopy to assess its architecture, and haematoxylin and eosin (H\&E), Sandiford and Alcian blue/periodic acid Schiff (ABPAS) stains were performed. The cases were assessed for extent of necrosis, inflammation, organisation and viral inclusions. On the Sandiford stain Gram-positive organisms are blue-black and Gramnegative organisms are red. The ABPAS stains neutral mucins and fungal walls bright red or magenta, and acid mucopolysaccharides blue.

Tissue samples from one patient were also ex-waxed for electron microscopy analysis.

\section{Results}

\section{Case 1}

A male infant born at 34 weeks' gestation, birth weight $1800 \mathrm{~g}$, underwent laparotomy and subsequent relook for midgut volvulus with extensive small-bowel necrosis on day 25 of life. Postoperatively he remained hypotensive and septic, and died the following day.

Blood cultures remained negative, but a pus swab from the peritoneum was positive for extended-spectrum beta-lactamaseproducing $K$. pneumoniae.

Histological examination of the resected bowel showed extensive, acute, full-thickness haemorrhagic coagulative necrosis with no significant inflammation or organisation. A prominent serosal biofilm (Fig. 1) comprising Gram-negative bacterial bacilli was highlighted on the Sandiford special stain within an acidic mucopolysaccharide 


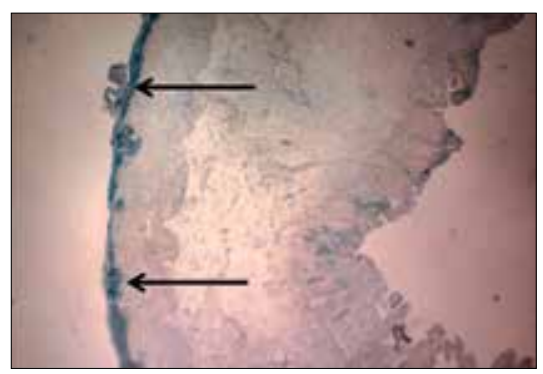

Fig. 1. Low-power view of the ABPAS stain, showing a blue biofilm along the serosal surface.

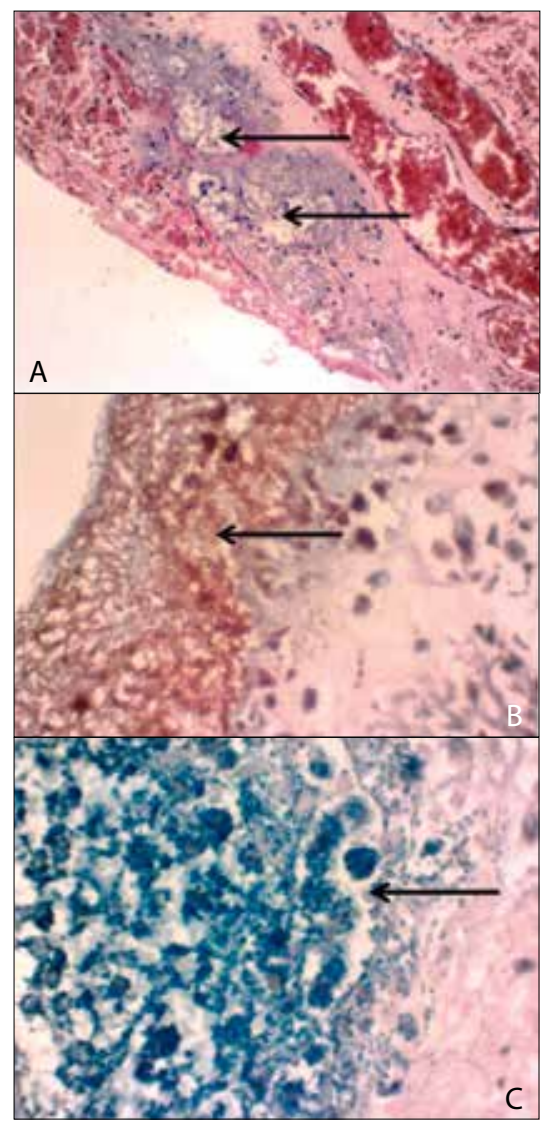

Fig. 2. (A) Medium-power view of the $H \& E$ stain, showing the mucoid colonies. (B) Highpower view of the Sandiford stain, showing the Gram-negative bacilli. (C) High-power view of the ABPAS stain, showing the bacilli surrounded by mucopolysaccharide (stains blue on the ABPAS stain).

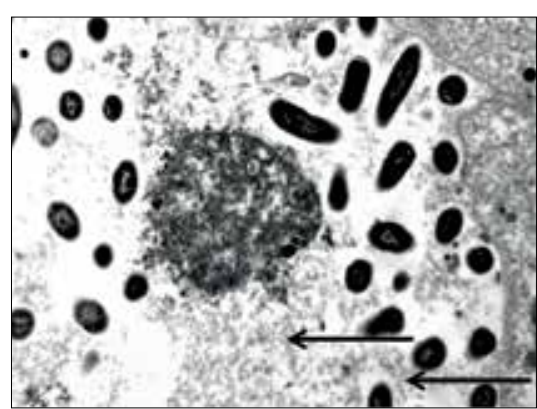

Fig. 3. Transmission electron microscopy showing bacterial bacilli surrounded by a matrix. matrix (ABPAS) (Fig. 2, A, B and C). The bacterial organisms were also present in the subserosal vessels, and similar scattered colonies were noted on the mucosal surface and intramurally. No viral inclusions, ova, parasites or fungal organisms were identified.

Transmission electron microscopy demonstrated the bacilli within a matrix consistent with mucopolysaccharide (Fig. 3).

\section{Case 2}

A male infant born at 28 weeks' gestation, birth weight $1180 \mathrm{~g}$, had a difficult neonatal course including respiratory distress due to hyaline membrane disease and NEC and was readmitted with sepsis, pneumonia, anaemia and oedema. He underwent laparotomy for abdominal distension due to a colonic stricture, and subsequent relook laparotomy showed extensive adhesions, exudative fibrin and multiple large pockets of pus. Necrotic bowel was resected. He remained unstable on inotropic and ventilator support and died 24 hours later with sepsis and multiorgan failure.

Blood culture always remained negative, but a pus swab from the peritoneum revealed abundant growth of multidrug-resistant K. pneumoniae.

Histological examination of the resected bowel, during the second procedure, showed transmural necrosis with an acute inflammatory response. There was adjacent organisation and regenerative epithelium. In addition, numerous colonies of Gramnegative bacilli within a mucoid matrix were present transmurally, highlighted on the Sandiford and ABPAS stains. Focal colonies within the submucosa appeared to be within vessels. No viral inclusions, ova, parasites or fungal organisms (ABPAS) were identified.

\section{Case 3}

A 3-day-old female infant born at 31 weeks' gestation, birth weight $1180 \mathrm{~g}$, underwent laparotomy and a subsequent relook for a focal mid-jejunal perforation. Postoperatively she remained septic with coagulopathy, thrombocytopenia, hyperglycaemia and acute kidney failure, and died on day 12 .

Blood culture and pus swab from the wound were positive for extended-spectrum beta-lactamase-producing Klebsiella.

Histological examination of the resected bowel, during the first procedure, showed areas of mucosal ulceration and perforation, with transmural necrosis and an organising acute peritoneal reaction. Focally, on the mucosal aspect, colonies of Gram-negative bacteria were noted within a mucopolysaccharide matrix. The viable mucosa showed superficial ischaemia and interstitial haemorrhage. In addition, abundant Gram-positive cocci were present (Sandiford stain).

Despite numerous blood cultures taken pre- and postoperatively, Klebsiella was identified on blood culture only once in case 3 .

In all cases, bacteriological cultures from pus swabs taken from the peritoneal cavity showed an abundant growth of a very resistant, extended-spectrum beta-lactamase K. pneumoniae species.

\section{Discussion}

The presence of biofilm formation in necrotic bowel has not been identified previously in infants, although it is well documented in septic patients with implanted medical and surgical devices, central venous and urinary catheters, and dental and chronic wound infections. ${ }^{[1,3,4]}$ Although the function of biofilms is unknown, their presence in necrotic bowel must have implications for the pathogenesis of bowel necrosis and for its medical and surgical management.

In our study, after conventional staining of a bowel specimen, excessive protein matrix was identified. The specimen was therefore subjected to biofilm specific staining. This led to further biofilm identification on subsequent specimens of necrotic bowel.

The exact process by which biofilmassociated micro-organisms can cause disease is speculative at present. ${ }^{[7]}$ Parsek and Singh, ${ }^{[6]}$ in 2003, suggested four criteria to define infections caused by biofilms:

- The infecting bacteria are adherent to some substratum or are surface associated.

- Direct examination of infected tissue shows bacteria living in cell clusters, or microcolonies, encased in an extracellular matrix. The matrix may often be composed of bacterial and host components.

- The infection is generally confined to a particular location. Although dissemination may occur, it is a secondary phenomenon.

- The infection is difficult or impossible to eradicate with antibiotics despite the fact that the responsible organisms are susceptible to killing in the planktonic state.

The encapsulated aggregations of organisms, which can form within hours, are highly resistant to antibiotic therapy and to systemic and local defence mechanisms. ${ }^{[8,9]}$ Biofilm infections are rarely resolved, and tissue adjacent to the biofilm may undergo 
collateral damage by immune complexes and invading neutrophils. ${ }^{[8]}$ The reasons for this are multifactorial, with changes in the microorganism, the development of resistant endotoxin formation and inability of antibiotics to diffuse through the biofilm's polysacchariderich matrix. ${ }^{[3,7]}$

Although K. pneumoniae is present as a saprophyte in the gastrointestinal tract, the identification of intramural Klebsiella biofilms has not been published in the English paediatric literature. ${ }^{[5,10]}$

The question of whether Klebsiella-associated biofilms were direct aetiological factors in causing bowel necrosis and the death of the patients reported on cannot be proven, but their presence satisfies all four of the Parsek and Singh postulates. ${ }^{[6]}$ We postulate that this sole offending organism shielded from environmental defence mechanisms could have played a part in the unfolding pathogenesis, either as a direct pathogen or promoter thereof.

The identification of Klebsiella biofilm in necrotic bowel has created a therapeutic dilemma. In K. pneumoniae, many studies have been performed in order to better highlight the mechanisms underlying this resistance, and have demonstrated that limitation of the penetration of antibiotic molecules through the biofilm matrix is not the main reason for the increasing resistance; rather it is the slow growth rate of Klebsiella spp. in the centre of the biofilm. In any case, other mechanisms are involved, and further studies are required to elaborate on new concepts in the preventive measures against nosocomial $K$. pneumoniae infections in the future. ${ }^{[1]}$ In chronic surface wounds with biofilms, the latter can be eradicated with topical bioflammacide therapy; this would not be an option for gastrointestinal disease. ${ }^{[12]}$

\section{Conclusion}

The identification of Klebsiella-associated biofilms in necrotic bowel of three infants in our institution has raised questions regarding the clinical implications of these biofilms. In future, all resected necrotic bowel should be investigated for presence of biofilms, and the clinical implications of a finding evaluated. If the presence of biofilm is confirmed in the majority of patients with bowel necrosis who died, this may represent a major therapeutic challenge.

1. Costerton JW, Stewart PS, Greenberg EP Bacterial biofilms: A common cause of persistent infections. Science 1999;284(5418):1318-1322. DOI:10.1126/science.284.5418.1318

2. Wilson M. Bacterial biofilms and human disease. Sci Prog 2001:84(Pt 3):235-254. DOI:10.3184/003685001783238998

3. Kennedy P, Brammah S, Wills E. Burns, biofilm and a new appraisal of burn wound sepsis. Burns 2010;36(1):49-56. DOI:10.1016/j.burns.2009.02.017

4. Dongari-Bagtzoglou A. Pathogenesis of mucosal biofilm infections: Challenges and progress. Exper Rev Anti Infect Ther 2008;6(2):201-208. DOI:10.1586/14787210.6.2.201

Von Rosenvinge EC, O'May GA, Macfarlane S, Macfarlane GT, Shirtliff ME. Microbial biofilms an gastrointestinal diseases. Pathog Dis 2013;67(1):25-38. DOI:10.1111/2049-632X.12020

Parsek MR, Singh PK. Bacterial biofilms: An emerging link to disease pathogenesis. Annu Rev Microbiol 2003;57(1):677-701. DOI:10.1146/annurev.micro.57.030502.090720

7. Donlan RM, Costerton JW. Biofilms: Survival mechanisms of clinically relevant microorganisms. Clin Microbiol Rev 2002;15(2):167-193. DOI:10.1128/cmr.15.2.167-193.2002

8. Stewart PS, Costerton JW. Antibiotic resistance of bacteria in biofilms. Lancet 2001;358(9276):135-138. DOI:10.1016/s0140-6736(01)05321-1

9. Hammond AA, Miller KG, Kruczek CI, et al. An in vitro biofilm model to examine the effect of Hammond AA, Miller KG, Kruczek CJ, et al. An in vitro biofilm model to examine the effect of
antibiotic ointments on biofilms produced by burn wound bacterial isolates. Burns 2011:37(2):312321. DOI:10.1016/i.burns.2010.09.017

10. Podschun R, Ullmann U. Klebsiella spp. as nosocomial pathogens: Epidemiology, taxonomy, typing methods, and pathogenicity factors. Clin Microbiol Rev 1998;11(4):589-603.

11. Vuotto C, Longo F, Balice MP, Donelli G, Varaldo PE. Antibiotic resistance related to biofilm formation in Klebsiella pneumoniae. Pathogens 2014;3(3):743-758. DOI:10.3390/pathogens 3030743

12. Phillips P, Wolcott R, Fletcher J, Schultz G. Biofilms made easy. Wounds international 2010. http:// www.woundsinternational.com/made-easys/view/biofilms-made-easy (accessed 12 January 2016).

Accepted 12 December 2015. 